\title{
Fiber Bragg Grating Inclinometer-enabled IoT Sensing System with Low Power Consumption and Small Size
}

\author{
Can Cui, ${ }^{1}$ Le Gao, ${ }^{2 *}$ Nina Dai, ${ }^{2 * *}$ and Qingshan $\mathrm{Xu}^{2}$ \\ ${ }^{1}$ School of Computer Science and Engineering, Chongqing Three Gorges University, Chongqing 404130, China \\ ${ }^{2}$ School of Electronics and Information Engineering, Chongqing Three Gorges University, \\ Chongqing 404130, China
}

(Received December 31, 2020; accepted April 28, 2021)

Keywords: fiber Bragg grating, inclinometer sensor, remote monitoring, subsidence, Internet of Things sensing

The application of fiber Bragg grating (FBG) sensors in the Internet of Things (IoT) has attracted wide attention for promising scenarios, such as remote monitoring of the subsidence of transformer substations, intelligent sensing of the power grid, and monitoring the safety of mines. However, disaster prewarning and forecasting in infrastructure construction require the key technology of remote sensing and monitoring. To resolve the current problems existing in monitoring the subsidence of substations built on a slope, an inclinometer based on FBG sensor technology is developed in this study. The FBG inclinometer sensor includes a rigid unit, a flexible sensing unit, and a connecting pin shaft. The flexible sensing unit is equipped with an FBG beam structure with uniform strength. The designed FBG is arranged symmetrically on the surface of the beam structure to eliminate the influence of temperature on measurement. In addition, a system that uses the FBG inclinometer sensor for remotely monitoring the subsidence of a transformer substation is designed. This system can display the subsidence of the substation on-site or at a remote terminal in real time. Indoor and field tests are carried out on the remote monitoring system. The test results show that the minimum resolution of the system is $0.1 \mathrm{~mm}$ and the maximum measurement error is less than $3 \%$, which meet the requirements of on-site use.

\section{Introduction}

In the Internet of Things (IoT), the exchange of information and communication are performed through cloud computing and related technologies. The IoT plays a critical role in modern IT by dealing with big data. In the IoT, optical-fiber-based sensing technology has a significant impact owing to its advantages. ${ }^{(1-3)}$ The International Telecommunication Union (ITU) pointed out that the IoT can connect everything in the world by using computer interconnection technology, radio frequency identification devices, sensor technology, wireless communication technology, and so forth. ${ }^{(4)}$ IBM has proposed the concept of a "smart Earth" that combines IoTs as a strategy for economic revitalization. ${ }^{(5)}$

\footnotetext{
*Corresponding author: e-mail: ag11509@163.com

** Corresponding author: e-mail: dainina83@163.com

Revised on July 7, 2021

https://doi.org/10.18494/SAM.2021.3281
}

ISSN 0914-4935 (C) MYU K.K. https://myukk.org/ 
The concept of "smart" in IT for infrastructure construction is expected to stimulate the economy, promote employment, and lead to a mature infrastructure platform. The action plan proposed by the European Commission (EC) clearly states that a large amount of financial support for the IoT will be provided at the technical level and suggests a network regulation plan at the government management level that is in accordance with existing regulations. ${ }^{(6)}$

In recent years, the continuous development of communication technology has contributed to sensing technology using optical fibers. ${ }^{(7,8)}$ Accordingly, fiber-optic sensors, e.g., functional fiber and non-functional fiber sensors, have been rapidly developed as they have advantages such as a small size, high sensitivity, strong anti-interference ability, and so forth. The sensors are widely applied in many fields including pharmaceutical manufacturing, shipping, and civil engineering. The development of fiber-optic sensors has enabled the rapid development of the IoT and made sensors increasingly important in applications. ${ }^{(9)}$

Fiber Bragg grating (FBG) sensors, as well as strain sensors, temperature sensors, acceleration sensors, displacement sensors, and so forth, demodulate specific wavelengths generated by external physical parameters. ${ }^{(10)}$ The demodulation system of an FBG sensor contains FBG filters, adjustable Fabry-Perot (F-P) interference filters, and a non-equilibrium Mach-Zehnder (M-Z) interferometer. Previous study results showed that the wavelength shift caused by strain and temperature changes is small, so accurate detection of the wavelength shift requires precise system measurement. Therefore, demodulation is a key technology in FBG sensors.

There are various FBG sensors for measuring strain, ${ }^{(11)}$ temperature, ${ }^{(12)}$ pressure, ${ }^{(13)}$ and so forth. These sensors are attracting much research interest owing to the increasing demand for the IoT. ${ }^{(14,15)}$ Traditional fiber-optic sensors measure light intensity or interference according to the sensor type. Light intensity sensors have problems caused by unstable light incidence, the loss of the signal through the optical fiber, and the aging of the detector. Interference-type sensors are used in a limited number of applications as they need two interference lights of equal intensity from fixed reference points. The modulation of the wavelength and the multiplexing capability of FBG sensors avoid such problems. FBG sensors are regarded as ideal for building diagnostics and the detection of impact, shape changes, and vibration damping. Thus, they are widely used in geodynamics, spacecraft, shipping, civil engineering, the power industry, medicine, and chemical sensing. FBG sensors can realize the direct measurement of temperature, strain, and other physical quantities. In addition, they have been divided into the reflective type and transmission type, with the reflective type, whose principle is shown in Fig. 1, more widely used.

The point-based optical fiber sensing technology is used in engineering as it detects the sensing signals at a designated point. A large-scale sensor network of fiber-optic point-based sensors has a high installation cost due to the scattered distribution of induction. When the sensors are not focused on the sources of the wavelength, they are not sufficiently sensitive to produce signals above the detection limit. Li et al. proposed a distributed fiber-optic sensor based on the Brillouin effect that solves the problem of point-based sensors. ${ }^{(16)}$ This sensor had the advantages of a small body, corrosion resistance, anti-electromagnetic interference, a wide transmission bandwidth, integrated networking of sensing and transmission, continuous monitoring, a low maintenance cost, affordability, rapid sensing, and high sensitivity. These 


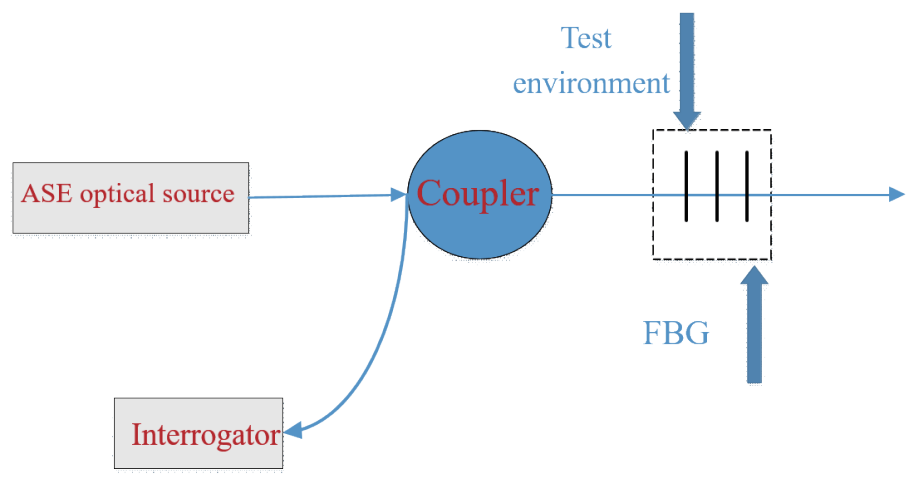

Fig. 1. (Color online) Schematic diagram of an FBG sensor (reflection type).

advantages allow it to be used to check the safety of pipelines, power cables, bridges, and missile systems and to diagnose buildings for subsidence and cracks. As point-based sensors are also used for detecting stress and strain in general, they are used in IoT networks in engineering, agriculture, security, and other fields. ${ }^{(17)}$

Monitoring the subsidence of a substation currently requires continuous measurement of the subsidence of a pile by manually measuring its level regularly. This reduces the efficiency of monitoring, generates inaccuracy caused by the limited amount of data, and makes it difficult to meet the required standard of subsidence monitoring. In recent years, FBG-based sensors have become used more widely as they are not affected by electromagnetic interference and have high sensitivity and accuracy, good insulation, and easy networking. Therefore, it is important to improve the theory and application of FBG-based sensors in subsidence monitoring systems used in transformer substations, intelligent power grid sensing systems, mine safety monitoring, and so forth.

In this study, we propose a technology based on fiber-optic sensing that improves the performance of gyroscopes, hydrophones, grating sensors, and current sensors. The proposed technology can be used for the FBG-sensor-based remote monitoring of subsidence at transformer substations. It also provides a new way of developing and manufacturing miniaturized IoT sensors with low power consumption, and enables the real-time display on site or at remote terminals.

\section{Design of Inclinometer Sensor Based on FBG}

An FBG-based inclinometer sensor is used to measure the relative angle of inclination between different locations caused by soil subsidence. It is composed of a rigid unit, a flexible sensing unit, and a connecting pin shaft as shown in Fig. 2. The rigid unit rotates around the connecting pins, and the flexible sensing unit measures the relative inclination angle at the position connecting the rigid unit.

The angle is determined by calculating the deflection (i.e., the strain) of the sensing unit in the sensor and using the geometric relationship. The flexible sensing unit is designed to have a 

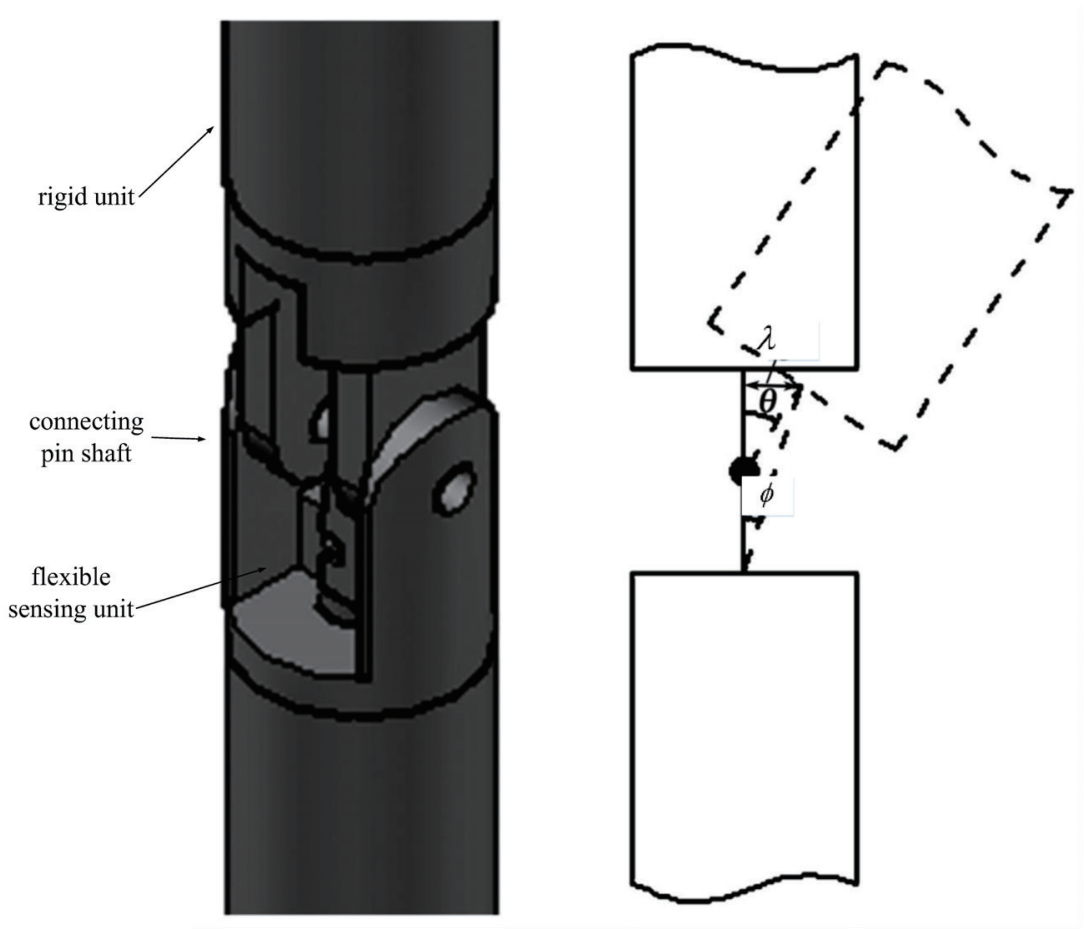

Fig. 2. Schematic diagram of an FBG-based inclinometer sensor.

beam structure with uniform strength. The structure is simple and easy to fabricate, and even a small load applies a large strain to it, which improves the precision and resolution of the sensor. When the rigid element has a relative tilt angle $\theta$ and the angle of the iso-strength beam of the sensing element is $\phi, \theta=2 \phi$ according to the geometric relationship. Therefore, the sensor has a magnifying effect in the measurement of the subsidence, which is described by the following relation involving the sine function of the inclination angle:

$$
\sin \theta=\frac{\lambda}{(l / 2)}
$$

where $l$ is the length of the beam of constant strength, and $\lambda$ is the free-end deflection of the beam of constant strength, which is calculated by using the cantilever beam model. Assuming that the fixed end width of the uniform-strength beam is $b_{0}$, its thickness is $h_{0}$, the free end of the uniform-strength beam concentrates the force $F$, and the elastic modulus is $\Delta$, then the bending strain $\varepsilon$ at any point on the surface of the beam is defined as follows:

$$
\varepsilon=\frac{6 F \cdot l}{\Delta \cdot b_{0} \cdot h_{0}^{2}}
$$

The deflection of the free end of the uniform-strength beam is defined as 


$$
\lambda=\int \frac{M(x)}{\Delta \cdot l} \cdot \frac{\partial M(x)}{\partial F} d x=\frac{6 F \cdot l^{3}}{\Delta \cdot b_{0} \cdot h_{0}^{3}} .
$$

Substituting Eqs. (2) and (3) into Eq. (1) gives

$$
\sin \theta=\frac{2 l}{h_{0}} \varepsilon .
$$

The bending strain of the uniform-strength beam is measured by an FBG-based inclinometer sensor that is placed symmetrically at the midpoint between the upper and lower surfaces of the beam. The strain is equal at all points on the beam surface, which prevents a measurement error from being generated by the position of the sensor and the chirping caused by the uneven distribution of stress. The factors that affect the wavelength sensing are bending and axial strains, and changes in temperature. The two wavelengths of the FBG-based inclinometer sensor are calculated as

$$
\begin{aligned}
& \Delta \lambda_{1}=\Delta \lambda_{M^{+}}+\Delta \lambda_{N}+\Delta \lambda_{T}, \\
& \Delta \lambda_{2}=\Delta \lambda_{M^{-}}+\Delta \lambda_{N}+\Delta \lambda_{T},
\end{aligned}
$$

where $\Delta \lambda_{M^{+}}$and $\Delta \lambda_{M^{-}}$are the bending strains of the upper and lower surfaces, respectively, $\Delta \lambda_{N}$ represents the axial strain, and $\Delta \lambda_{T}$ is the wavelength change caused by a change in temperature. $\Delta \lambda_{M^{+}}$and $\Delta \lambda_{M^{-}}$are equal in magnitude but have opposite directions. The inclination angle of the sensor is only related to the bending strain. According to Eqs. (5) and (6), the influence of the axial strain and temperature is eliminated by the following equation:

$$
\varepsilon=\frac{\Delta \lambda_{1}-\Delta \lambda_{2}}{2 \cdot k_{\varepsilon}}
$$

Thus, the temperature of the sensor is self-compensated, so the test result becomes accurate owing to the doubled variation of the wavelength. The relation between the inclination angle and the center wavelength of the FBG-based inclinometer sensor is defined as

$$
\sin \theta=\frac{l}{k_{\varepsilon} h_{0}} \cdot\left(\Delta \lambda_{1}-\Delta \lambda_{2}\right)
$$

When $\theta$ approaches $\sin \theta$ as the angle decreases, the relationship between the inclination angle and the wavelength offset of the sensor becomes approximately linear. The parameters $l$ and $h$ of the sensing element are determined to obtain the linear coefficient for calibration in practical application. 


\section{Subsidence Monitoring with FBG-based Inclinometer Sensor}

\subsection{Measurement principle of FBG-based inclinometer sensor}

Figure 3 describes the measurement principle of the FBG-based inclinometer sensor. When incident light propagates along the FBG and satisfies the wavelength of the Bragg grating, the light is reflected to create a spectrum with a single peak. Otherwise, the spectrum has multiple peaks.

The central wavelength of the reflected light is called the fiber Bragg wavelength and is calculated as follows:

$$
\frac{d \lambda_{F B G}}{\lambda_{F B G}}=K_{T} \cdot d T+K_{M} \cdot d M
$$

where $\lambda_{F B G}$ is the wavelength of the FBG, $K_{T}$ is the temperature sensitivity coefficient, $T$ is the temperature, $K_{M}$ describes the strain sensitivity coefficient, and $M$ is the strain.

Equation (9) reveals that the rate of change of the FBG wavelength is related to the temperature and strain. When the FBG is affected by external stress to produce strain, the strain changes the effective refractive index of the grating, which results in a change in the FBG wavelength. The change in the FBG wavelength under the influence of the external temperature, thermal expansion, and cold contraction causes the effective refractive index of the FBG to change. Thus, the strain information is obtained in real time if the FBG wavelength is measured with the sensors and calibrated using the temperature.

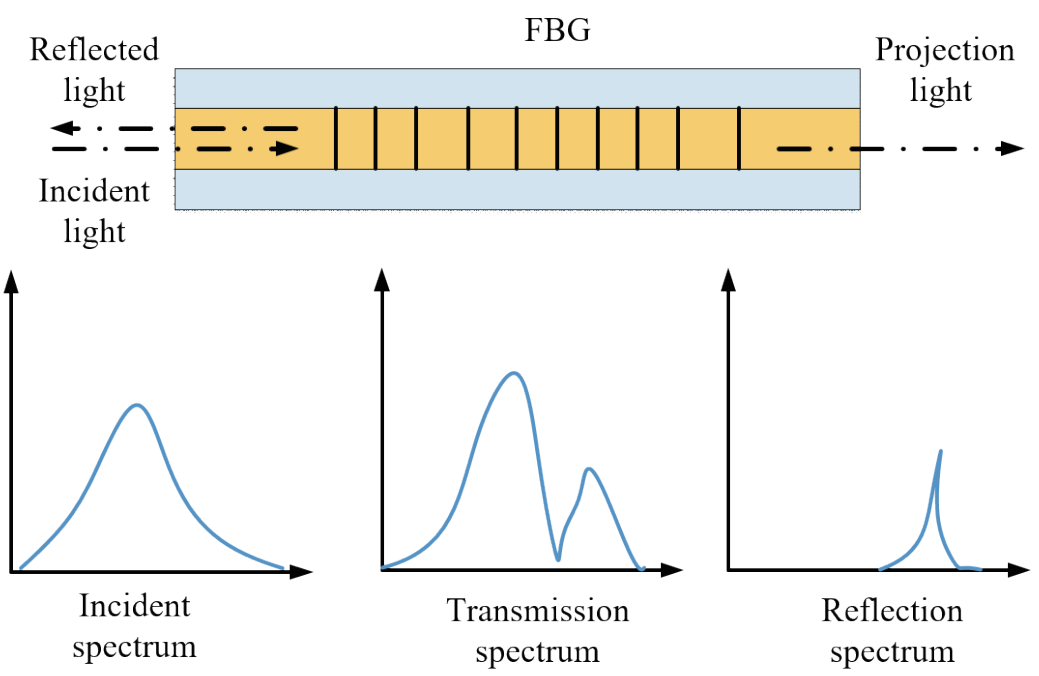

Fig. 3. (Color online) Measurement principle of an FBG-based inclinometer sensor. 


\subsection{Principle of subsidence monitoring}

Figure 4 is a schematic diagram of the designed FBG-based inclinometer sensor. The sensor is composed of a monitoring pile, a wedge plate, a counterweight block, a push rod, and an FBG. The counterweight block is placed on the ground, and the wedge plate is fixed on the counterweight block. The push rod, employing a flat pin shaft, is fixed with a monitoring pile to ensure that the push rod moves only horizontally.

When subsidence of the soft surface soil occurs, the counterweight block will drive the wedge to subside simultaneously. Then, the wedge plate moves the compressed push rod horizontally, thus further compressing the FBG to generate strain and convert the displacement of the surface into the subsidence of the FBG. The measurement process is based on the following equations:

$$
\begin{aligned}
& \tan \theta=\frac{x}{d}, \\
& w=\frac{F l^{3}}{48 E I}, \\
& x=w,
\end{aligned}
$$

where $\theta$ is the angle between the wedge plates, $x$ is the horizontal displacement of the push rod, $d$ is the displacement of the surface, $w$ is the deflection of the fiber grating, $F$ is the thrust received by the FBG, $l$ is the length of the FBG, $E$ is the elastic modulus of the FBG, and $I$ is the inertia of the FBG section. Combining the three equations in Eq. (10) gives

$$
d=\frac{F \cdot l^{3}}{48 E \cdot I \cdot \tan \theta}
$$

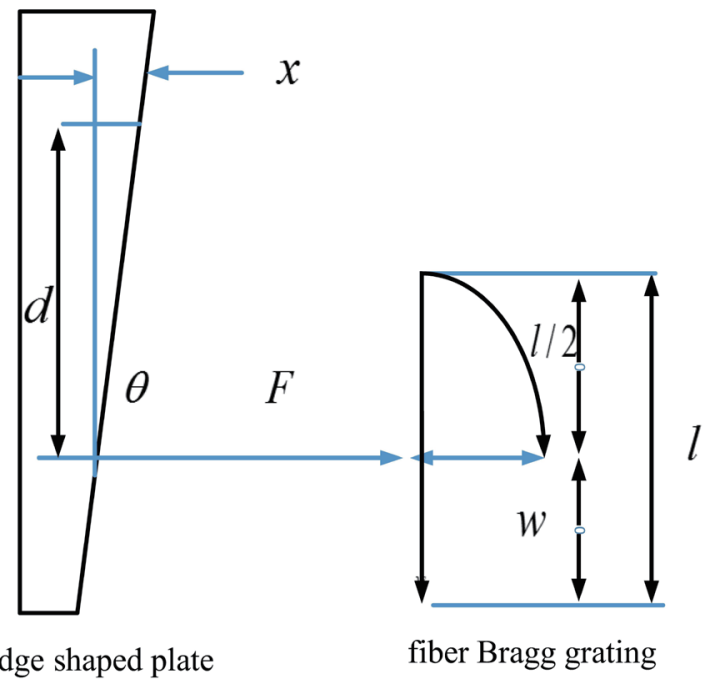

Fig. 4. (Color online) Schematic diagram of the designed FBG-based inclinometer sensor. 
where $F$ is measured by the FBG and $l, E, I$, and $\theta$ are constants. The measured values of the constants and the FBG are substituted into Eq. (11) to measure the displacement in real time.

A field test was performed on a transformer substation to evaluate its performance in remotely monitoring subsidence. In the test, the acquired signal is first transmitted through the optical fiber, then passes through a linear actuator, and finally arrives at the FBG sensor. The field test for the FBG sensor-based inclinometer is shown in Fig. 5.

\subsection{Calculation of subsidence}

The program for calculating the displacement was written in LabVIEW (National Instruments). ${ }^{(18)}$ The created software interface is shown in Fig. 6. The interface has three functions: waveform display, function buttons, and filtering. The waveform display shows the waveforms of the measured subsidence displacement data in real time. The abscissa of the waveform shows the time and the ordinate shows the subsidence displacement. The data of up to 15 measuring points are displayed in real time. The function buttons are used for saving location information and starting/stopping the operation. The filtering function filters the data collected from the sensors in real time.

\section{Results and Discussion}

After developing the system for remotely monitoring subsidence, indoor tests to validate the system were carried out by using a standard displacement transducer (LP801-150 by OMEGA). Figure 7 shows the results of the indoor tests of the monitoring system at different temperatures. The measurement error was less than $3 \%$ and the minimum resolution was $0.1 \mathrm{~mm}$, which meet the requirements for on-site measurement. ${ }^{(19)}$

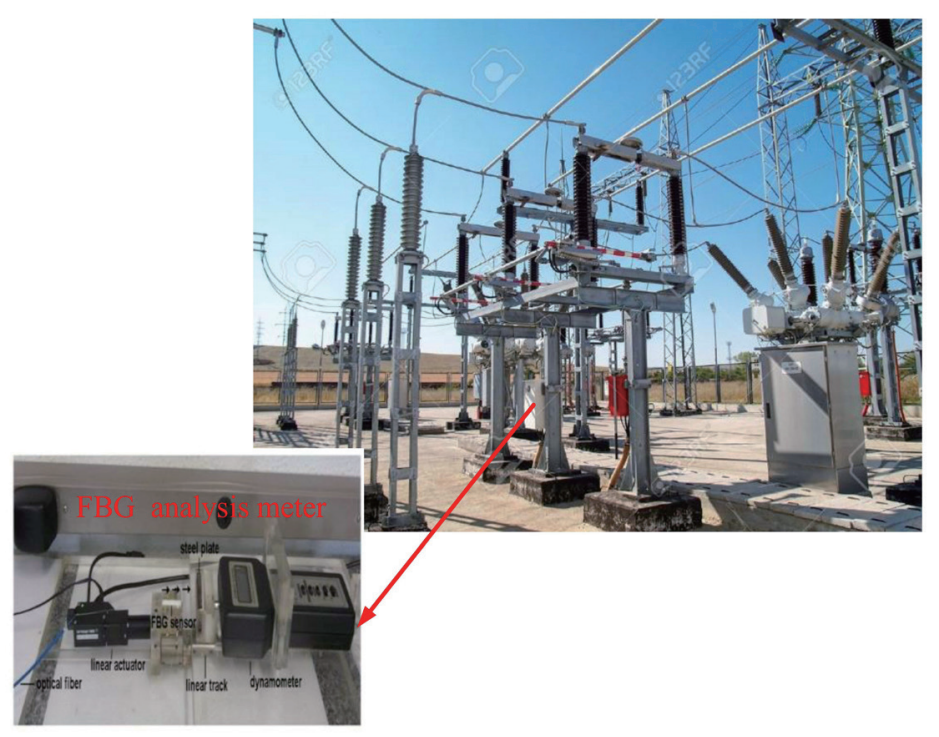

Fig. 5. (Color online) Field test. 


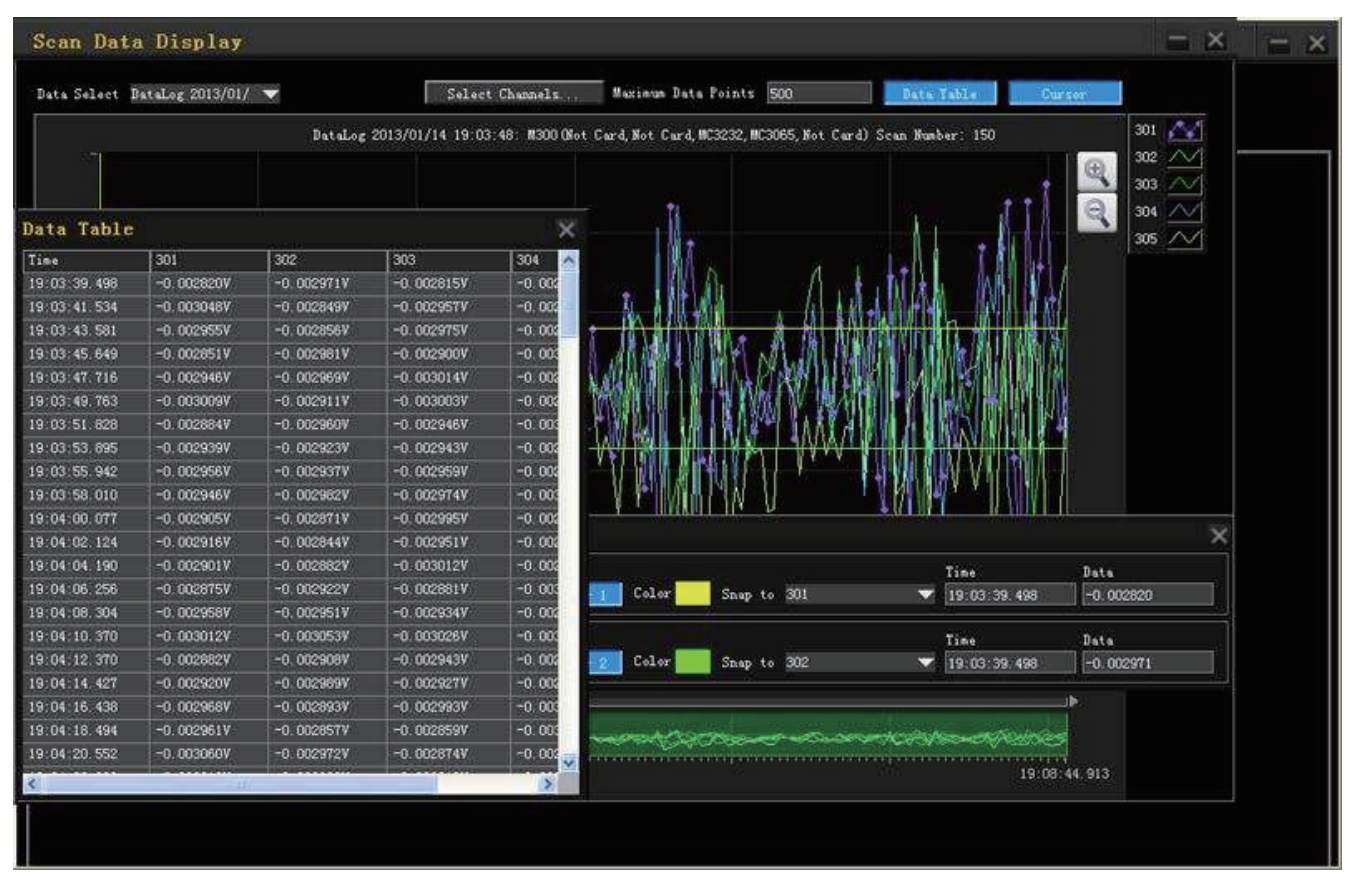

Fig. 6. (Color online) Interface of the calculation software.

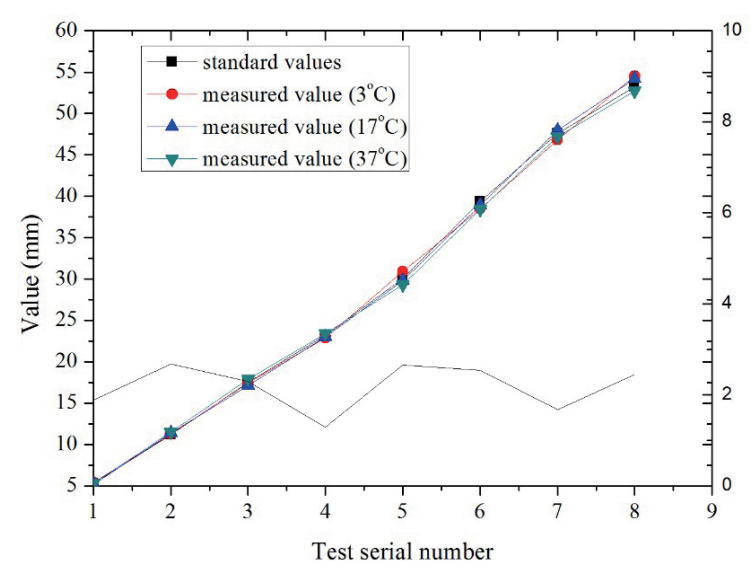

Fig. 7. (Color online) Result of indoor tests of the remote monitoring system with the FBG-based inclinometer sensor.

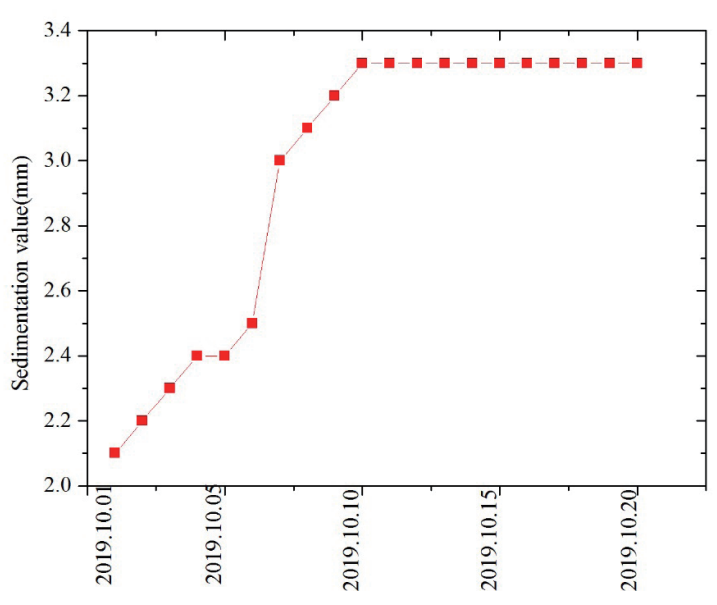

Fig. 8. (Color online) Results of field test of the remote monitoring system.

After the indoor tests, multiple field tests were conducted. The setup of one of the field tests is shown in Fig. 5 and the results of the field tests are shown in Fig. 8. The subsidence data at a single measuring point between October 1 and 20, 2019 were recorded. The displacements due to subsidence between October 1 and 6 were smaller than the later measurements. Displacement of over $1 \mathrm{~mm}$ was observed on October 10. The displacement increased as the backfilled layer settled slowly over a short period and eventually became stable after the filling of the foundation 
pit in the transformer substations. The results coincide with the expected subsidence of the foundation pit, indicating that the system is suitable for measuring the subsidence of transformer substations on an inclined slope.

\section{Conclusions}

Owing to the shortcomings of current substation subsidence monitoring technology, a kind of inclinometer based on FBG sensor technology is proposed. The proposed sensor includes a rigid unit, a flexible sensing unit equipped with an FBG, and a connecting pin shaft. Temperature compensation was realized by arranging the FBG symmetrically on the beam surface of the sensor. When the slope deforms, the rigid element then deflects at the connecting pin and is offset by the FBG wavelength in the flexible sensing element. According to the linear relationship between the central wavelength of the FBG and the strain and angle, the relative inclination angle between the rigid elements is obtained and the displacement is calculated through the geometric relationship, which can realize the monitoring of soil displacement. We developed a system for remotely monitoring displacement due to subsidence using an FBGbased inclinometer sensor that enables the real-time acquisition of subsidence information. The data can be transmitted from on-site sensors to the monitoring station through either cable transmission or a general packet radio service (GPRS) network. Software to process the data, display the wavelengths, and calculate the displacement was developed. Indoor and field test results showed that the resolution of the system was $0.1 \mathrm{~mm}$ and the measurement error was less than $3 \%$, which meet the requirements of field tests.

\section{Acknowledgments}

This work was supported by the Science and Technology Project of Chongqing Education Commission (Nos. KJQN202001223 and KJQN2019012) and Chongqing Three Gorges University (No. 19QN08).

\section{References}

1 D. Vigneswaran, M. S. Mani Rajan, B. Biswas, and K. Ahmed: Wire. Netw. 26 (2020) 3217. https://doi. org/10.1007/s11276-019-02153-w

2 M. F. Domingues, N. Alberto, C. S. J. Leitao, C. Tavaras, E. R. de Lima, A. Radwan, V. Sucasas, J. Rodiriguez, P. S. B. Andre, and P. F. C. Antunes: IEEE Internet Things J. 6 (2019) 207. https://doi.org/10.1109/ JIOT.2017.2723263

3 Z. J. Ke, D. L. Tang, X. Lai, Z. Y. D, and Q. Zhang: Optik 157 (2018) 1094. https://doi.org/10.1016/j. ijleo.2017.11.130

4 J. Gubbi, R. Buyya, S. Marusic, and M. Palaniswami: Future Gener. Comput. Syst. 29 (2013) 1645. https://doi. org/10.1016/j.future.2013.01.010

5 K. Aberer, G. Alonso, and D. Kossmann: Sigmod Rec. 35 (2006) 40. https://doi.org/10.1145/1228268.1228277

6 N. C. Swayze, W. T. Tinkham, J. C. Vogeler, and A. T. Hudak: Remote Sens. Environ. 263 (2021) 112540. https://doi.org/10.1016/j.rse.2021.112540

7 V. Kaur and S. Singh: Opt. Fiber Technol. 48 (2019) 159. https://doi.org/10.1016/j.yofte.2018.12.015

8 C. Cheng, Z. Yang, Y. Ou, Z. Chen, J. Chen, and H. Lu: Opt. Fiber Technol. 48 (2019) 303. https://doi. org/10.1016/j.yofte.2019.01.032

9 W. Zhang and D. J. Webb: IEEE Photon. Technol. Lett. 31 (2019) 234. https://doi.org/10.1109/LPT.2018.2889758 
10 H. Zhou, Z. Pan, Z. Liang, C. Zhao, Y. Zhou, and F. Wang: KSCE J. Civ. Eng. 23 (2019) 1911. https://doi. org/10.1007/s12205-019-0787-6

11 H. Zhang, G. Shen, and D. Jin: Adv. Mat. Res. 225-226 (2011) 212. www.scientific.net/AMR.225-226.212

12 C. Rodrigues, C. Felix, A. Lage, and J. Figueiras: Eng. Struct. 32 (2010) 1993. https://doi.org/10.1016/j. engstruct.2010.02.033

13 A. S. Allil, F. da Silva Dutra, A. Dante, C. C. Carvalho, R. C. da Silva Barros Allil, and M. M. Werneck: IEEE Tran. Instrum. Meas. 11 (2020) 1. https://doi.org/10.1109/TIM.2020.3014751

14 J. Yu and D. Liang: Opt. Fiber Technol. 49 (2019) 7. https://doi.org/10.1016/j.yofte.2019.01.024

15 Y. Liu, Z. Jiang, R. Badcock, J. Fang, X. Yan, W. Chen, X. Fang, Z. Zhang, and W. Zhou: IEEE Trans. Appl. Supercond. 30 (2020) 1. https://doi.org/10.1109/TASC.2020.2990382

16 Z. Li, G. Hou, K. Wang, and J. Hu: Opt. Fiber Technol. 61 (2021) 102446. https://doi.org/10.1016/j. yofte.2020.102446

17 Y. C. Manie, P.-C. Peng, R.-K. Shiu, R.-K. Shiu, Y.-T. Hsu, Y.-Y. Chen, G.-M. Shao, and J. Chiu: J. Light. Technol. 38 (2020) 6. https://doi.org/10.1109/JLT.2020.2971240

18 P. Lu, Y. Fan, W. Pan, and D. Cao: J. Phys.: Conf. Ser. 1449 (2020) 012072. https://doi.org/10.1088/17426596/1449/1/012072

19 J. Hu, W. Wang, Z. Zhang, K. Zhang, K. Hua, and R. Chen: Int. J. Emerging Electr. Power Syst. 20 (2019) 20180215. https://doi.org/10.1515/ijeeps-2018-0215

\section{About the Authors}

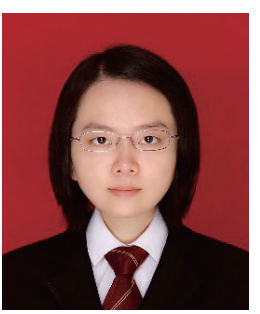

Can Cui received her bachelor's degree in communication engineering from Southwest University and her M.S. degree in agricultural engineering and information technology from Chongqing Three Gorges University, China. She has worked at Chongqing Three Gorges University since 2020 as a teaching assistant. Her research interests include the Internet of Things, fiber Bragg gratings, and infrared energy transmitting fibers.(2016017@sanxiau.edu.cn)

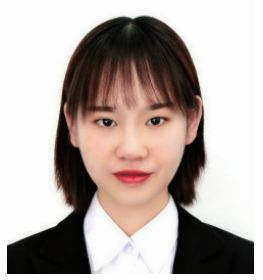

Le Gao received her bachelor's degree in electronic information science and technology from Sichuan University of Light and Chemical Engineering. She is studying electronic science and technology related to electric vehicles in the graduate school of Chongqing Three Gorges University. Her research interest lies in the key technologies of electric vehicles and vehicle network communication.(ag11509@163.com)

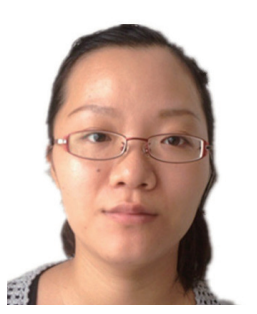

Nina Dai received her B.S. degree in electronic information engineering and her M.S. degree in circuits and systems from Central China Normal University in China. She joined Chongqing Three Gorges University in 2008, where she is an associate professor. Her research interests include communication and coding technology. (dainina83@163.com)

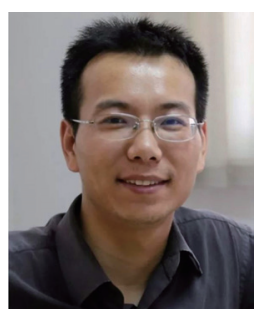

Qingshan Xu received his M.S. degree in electrical engineering from Hehai University and his Ph.D. degree in electrical engineering from Southeast University. He joined Chongqing Three Gorges University in 2018, where he is a professor. Her research interests include ubiquitous Internet of Things communication technology. (A58238735@outlook.com) 